\title{
Perfil Microbiológico das Precauções de Transmissão nas Internações e nos CTIs em Hospital Universitário de Canoas-RS, de maio/2011 a maio/2014
}

\author{
Sobreira, L.S.; Tonin, J.J.; Coronel, J.L.; Zambon, J.D.S.; Roza, L.E.M.; \\ Amaral, M.V.F e Vidaletti, T.B.;
}

Apresentador: Lucas Sobreira

\section{Resumo}

Introdução: O objetivo deste estudo é traçar o perfil dos microorganismos dos isolamentos em Hospital Universitário de Canoas, Rio Grande do Sul. Método: Estudo analítico retrospectivo não intervencional, avaliando perfil dos germes em precauções de transmissão de maio/2011 a maio/2014. Utilizou-se o banco de dados do Sistema de Controle de Infecções Hospitalares. Para a análise estatística foi utilizado o programa IBM SPSS Statistics V.20. Foram utilizados Análise Estatística Descritiva e Crostables para demonstrar dados dos germes, sítios de coleta de amostra e setores. Resultado: Houve 1037 precauções de transmissão, com os germes: Acinetobacter SPP com 29,6\% (307), Klebsiella Carba Resistente 8,7\% (90), Klebsiella Pneumoniae ESBL 7,1\% (74), Tuberculose 5,6\% (58), MRSA 5,3\% (55), Pseudomonas Aeruginosa Carba Resistente 4,9\% (51), Pseudomonas Aeruginosa 3,9\% (40), VRE 3,8\% (39), Klebsiella Pneumoniae Carba Resistente KPC POSITIVO 3,5\% (36), E. Coli ESBL 2,7\% (28), Klebsiella Pneumoniae ESBL 2\% (21) e outros germes com menos de $2 \%$ cada. Por setor, identificamos $61 \%$ no CTI Adulto, 26,9\% na Internação, $8,6 \%$ na CTI Pediátrica e 3,6\% na CTI Neonatal. Principais germes na CTI Adulto são Acinetobacter
SPP com 21,6\%, Klebsiella Pneumoniae Carba Resistente 8,4\% e Pseudomonas Aeruginosa Carba Resistente 4,1\%. No setor de internação Acinetobacter SPP. 6,8\%, Klebsiella Pneumoniae ESBL $4,7 \%$ e MRSA e Tuberculose com $2 \%$ cada. No CTI Pediátrico Klebsiella Pneumoniae 1,5\%, Acinetobacter SPP 1,3\% e VRE 1,6\%. Na CTI Neonatal Staphyllococcus Epidermidis e Serratia SPP com $0,6 \%$ cada e Klebsiella Pneumoniae com $0,4 \%$. Os sítios de coleta da amostra demonstraram, em ordem de infecção: Aspirado traqueal 25,3\% com Acinetobacter SPP, Pseudomonas Aeruginosa Carba Resistente e Klebsiella Pneumoniae (13,2\%, $2,8 \%$ e $2 \%$ respect.) Urina 19,3\% com Klebsiella Pneumoniae Carba Resist., Klebsiella Pneumoniae Ertapenen Resist.e VRE (3,9\%, 3,6\% e 2\% respectivamente) Hemocultura 12,6\% com Acinetobacter SPP, Klebsiella Pneumoniae ESBL e MRSA (4,8\%, 2,1 e 1,4\% respect.). Conclusão: Conhecimento sobre a epidemiologia de microrganismos nosocomiais e suas peculiaridades são fundamentais para elaborar ações contra os germes. Conhecer a prevalência dos germes locais pode otimizar a conduta médica, aliando a prática clínica à robustez da estatística.

\section{Referência:}

Sobreira, L.S.; Tonin, J.J.; Coronel, J.L.; Zambon, J.D.S.; Roza, L.E.M.; Amaral, M.V.F e Vidaletti, T.B.;. Perfil Microbiológico das Precauções de Transmissão nas Internações e nos CTIs em Hospital Universitário de Canoas-RS, de maio/2011 a maio/2014. In: II Congresso Brasileiro de Medicina Hospitalar - II CBMH [= Blucher Medical Proceedings, vol.1, num.5] São Paulo: Editora Blucher, 2014. p.10 